\title{
Effect of particle roughness on the bulk deformation using coupled boundary element and discrete element methods
}

\author{
Sadegh Nadimi ${ }^{1,2}$ (D) Ali Ghanbarzadeh ${ }^{1} \cdot$ Anne Neville$^{1} \cdot$ Mojtaba Ghadiri $^{1}$
}

Received: 26 February 2019 / Revised: 19 August 2019 / Accepted: 30 September 2019 / Published online: 11 October 2019

(c) The Author(s) 2019

\begin{abstract}
Particles slide and roll on each other when a granular medium is sheared. Consequently, the tribological properties, such as inter-particle friction and adhesion, play a major role in influencing their bulk failure and rheology. Although the influence of roughness on adhesion and friction of contacting surfaces is known, the incorporation of the surface roughness in the numerical modelling of granular materials has received little attention. In this study, the boundary element method (BEM), which is widely used for simulating the mechanics of interacting surfaces, is coupled with discrete element method (DEM) and the bulk deformation of granular materials is analysed. A BEM code, developed in-house, is employed to calculate the normal force-displacement behaviour for rough contact deformations, based on which a contact model is proposed. This is an efficient and relatively fast method of calculating the contact mechanics of rough surfaces. The resulting model is then implemented in the simulations by DEM to determine the effect of micro-scale surface roughness on the bulk compression of granular materials. This study highlights the importance of the effect of surface characteristics on contact behaviour of particles for the case of shallow footing and provides an efficient approach for modelling the flow behaviour of a large number of rough particles.
\end{abstract}

Keywords Contact mechanics $\cdot$ Numerical modelling $\cdot$ Discrete element method $\cdot$ Boundary element method

\section{List of symbols}

$\mathrm{A}_{\mathrm{c}} \quad$ Area of contact

E Elastic modulus

$E^{*} \quad$ Effective contact elastic modulus

$\mathrm{H}$ Hardness

$\mathrm{N} \quad$ Total number of nodes in the domain

$\varphi \quad$ Coefficient of friction

$p\left(s_{1}, s_{2}\right) \quad$ Applied contact pressure

$\mathrm{r} \quad$ Rigid body movement of two rough surfaces in normal direction

$R \quad$ Radius of sphere

$\left(s_{1}, s_{2}\right) \quad$ Coordinate of area that pressure is applied

$\mathrm{S}_{\mathrm{q}} \quad$ Root mean squared height

$\overline{u_{z}}(\mathrm{x}, \mathrm{y}) \quad$ Material deformation

$F_{N} \quad$ Load in the normal direction

Sadegh Nadimi

Sadegh.Nadimi-Shahraki@ncl.ac.uk

1 Faculty of Engineering, University of Leeds, Leeds LS2 9JT, UK

2 Present Address: School of Engineering, Newcastle University, Newcastle upon Tyne NE1 7RU, UK $(\mathrm{x}, \mathrm{y}) \quad$ Coordinate in which surface deformation is obtained

$\mathrm{Z}_{1}, \mathrm{Z}_{2} \quad$ The surface profiles of the two rough particles

$\alpha, \beta \quad$ Roughness parameters

$\nu \quad$ Poisson's ratio

\section{Introduction}

The mechanical behaviour of granular materials originates from force transmission at inter-particle contacts [18]. The use of discrete numerical modelling approaches enables the calculation of forces acting on individual particles, which is a non-trivial task in experimental approaches. Since its introduction by Cundall and Strack [3], DEM has been widely used for the modelling of particle interactions within an assembly. In most of the works reported so far, spheres or assemblies of spheres are used to represent particulate shape in order to facilitate contact calculations and speed up the simulations. However, particulate solids commonly have complex shapes, asperities and diverse chemical and mineralogical compositions and structures. 
The importance of grain shape in force transmission has been highlighted in a number of studies (e.g. [1, 20, 25]) and several attempts have been made to include grain shape in DEM simulations (e.g. [4, 14]). Regarding asperities, it has experimentally been observed that the nano-scale surface roughness of particles influences the bulk material properties [23, 24, 27, 28]. However, there have been few attempts to include particle surface roughness in DEM. The works so far can be categorised into two groups: direct geometry refinement, and contact model enhancement $[1,12,23$, $31,32]$. In the former case, direct inclusion of roughness in DEM modelling has been carried out by assuming that asperities are small spheres, which are bonded to the main particles. This methodology is computationally expensive and restricted to surface roughness with a spherical shape $[12,31]$. For the latter, development of normal force-displacement relationships for rough surfaces has been conducted by Cavarretta et al. [1], Otsubo et al. [23], and Zhao and Feng [32] using the statistical approach of Greenwood and Tripp [10]. In these models, a particle-scale roughness index proposed by Greenwood et al. [11] has been used. Cavarretta et al. [1] have subdivided the deformation progression of rough particles into asperity-dominant contact, transitional and Hertzian contact. They identified a threshold force and displacement, where the contact response transits from the so-called 'initial plasticity' to a Hertzian response. They used single particle compression tests to confirm the applicability of Hertz analysis for the last stage of deformation when the normal force exceeds the threshold. The initial stage of the indentation on rough surfaces was reported to be plastic, and the displacement was modelled using the hardness and elastic modulus of the material as input parameters. Initial plastic displacement was measured macroscopically and the hardness was the macroscopic hardness of the material with no means of considering asperities. The effect of loading on displacement of individual asperities could not be explored, since there was no means of reflecting shape of micro-asperities in the contact mechanics calculations. Therefore, the model has been further developed by Otsubo et al. [23] in which the threshold force and displacement are based on the assumptions of Greenwood and Tripp [10].

Deterministic contact mechanics gives the flexibility of considering complex surface characteristics in contact calculations. In tribology, there have been extensive efforts in investigating the effect of roughness on contact mechanics, covering both experimental and modelling aspects. In this study, an attempt is made to develop a force-displacement relationship for rough surfaces by coupling boundary element method (BEM) and DEM modelling. The contact behaviour of rough particles is analysed by BEM and the force-displacement relationship for rough surfaces is obtained and used in DEM calculation. The surface roughness and mechanical properties of glass ballotini (as used by previous researchers, e.g. [23]) are inputted in BEM and the output is represented by a customised contact model in DEM simulations. Details of BEM and DEM simulations are described below.

\section{Contact mechanics modelling}

\subsection{Boundary element method (BEM)}

The normal force-displacement relationship for the contact of rough particles is modelled using an in-house contact mechanics code developed by Ghanbarzadeh et al. [7, 8]. When two rough surfaces are in contact, individual asperities will bear the load and the area of contact is different from nominal Hertzian one. In order to calculate the discontinuous patchy real contact area and the contact pressure, the energy of the interface should be minimised [16]. The deformation of the interfaces $\bar{u}_{z}(\mathrm{x}, \mathrm{y})$ is then calculated using Boussinesq-Cerruti theory:

$\bar{u}_{z}=K * p_{d}=\int_{-\infty}^{+\infty} \int_{-\infty}^{+\infty} K(x-\xi, y-\eta) p(\xi, \eta) d \xi d \eta$

$p(\xi, \eta)$ is the applied load, $K$ is the convolution kernel and is calculated using half-space approximation:

$K(x-\xi, y-\eta)=\frac{1}{\pi E^{*}} \frac{1}{\sqrt{(x-\xi)^{2}+(y-\eta)^{2}}}$

where $E^{*}$ is the equivalent elastic modulus of both materials $\left(\frac{1}{E^{*}}=\frac{\left(1-v_{1}^{2}\right)}{E_{1}}+\frac{\left(1-v_{2}^{2}\right)}{E_{2}}\right)$. Here, $v_{1}, v_{2}, E_{1}$ and $E_{2}$ are Poisson's ratio and elastic modulus of materials 1 and 2 respectively.

By movement of the rigid body in the normal direction, the interference $(i)$ between the contacting surfaces can be obtained (Fig. 1). For the nodes experiencing contact, the elastic deformation must be equal to the body interference and the pressure is generated at the asperity. The summation of the contact forces arising from local nodes must also be

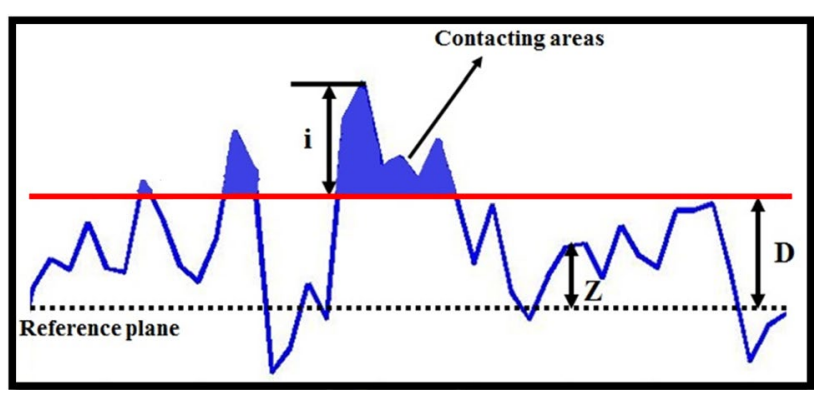

Fig. 1 Schematic of the contact of rough surfaces 
equal to the applied load. Therefore, the set of equations for the contact of rough surfaces are as follows:

$\left\{\begin{array}{lr}r=\left(Z_{2}(x, y)-Z_{1}(x, y)\right)+\bar{u}_{z}(\mathrm{x}, \mathrm{y}) & \forall x, y \in A_{c} \\ p(x, y)>0 & \forall x, y \in A_{c} \\ p(x, y)<H & \\ \sum_{i, j=N} p_{i, j}=F_{N} & \end{array}\right\}$

In Eq. $2, r$ is the rigid body movement of two rough surfaces in the normal direction, $Z_{2}$ and $Z_{1}$ are the surface profiles of the two rough particles, $F_{N}$ is the total applied load in the normal direction, $H$ is the hardness of the softer material in contact, $A_{c}$ is the area of contact and $N$ is the total number of nodes in the domain of study. In the current contact mechanics model, an elastic-perfectly plastic approach is incorporated. Hardness of the softer material is set to be the threshold for the plastic flow and the pressure does not exceed this value. This approach is widely used in other works in order to simulate elastic-plastic contact deformation [30]. More details of the approach can be found in Sahlin et al. [26] and Ghanbarzadeh et al. [7, 8].

\subsection{Rough surface generation and analysis}

Digitised rough surfaces are necessary inputs of the contact mechanics simulation. They are generated using the method introduced by $\mathrm{Hu}$ and Tonder [15], using 2-D digital filters and autocorrelation functions. Fast Fourier Transforms are used for numerical efficiency. An autocorrelation function defines the shape of the asperities. There are several parameters that can be used to characterise the topography of the surfaces, such as Root Mean Square (RMS) roughness, skewness, kurtosis etc. Incorporation of all these surface parameters in a digitised surface needs careful characterisation of the real surfaces for extracting the desired parameters as input to the surface generation models [2]. Therefore, for simplicity only RMS roughness of the surfaces is used in the numerical simulations as input parameter and the results of all different cases are compared with the smooth case. An example of the surfaces used in the numerical simulation is shown in Fig. 2. The upper surface is set to be perfectly smooth and rigid and the lower surface has the composite surface roughness $\left(S_{q}^{*}\right)$ of both particles in contact. The $S_{q}^{*}$ is defined by Eq. 4. The contact in this condition is known to be equivalent to the real system of two rough particles.

$S_{q}^{*}=\sqrt{S_{q 1}^{2}+S_{q 2}^{2}}$

where $\mathrm{S}_{\mathrm{q} 1}$ and $\mathrm{S}_{\mathrm{q} 2}$ are the RMS roughness values of particles 1 and 2 , respectively.

\subsection{Normal force-displacement relationship}

In order to obtain the normal force-displacement curves, the upper particle (upper surface) is moved in the normal direction. The normal force $\left(F_{N}\right)$ is then calculated from the contact mechanics code solving the set of Eq. 2. Movement of the upper surface is adjusted by changing the value of $r$ in Eq. 2. The deformation of the particles is simulated with step-wise increase in the value of $r$ and the corresponding normal force is recorded. The results of normal force-displacement are compared with the Hertzian deformation for the case of entirely smooth particles $\left(S_{\mathrm{q}}=0 \mu \mathrm{m}\right)$ in order
Fig. 2 Numerical model used in this study

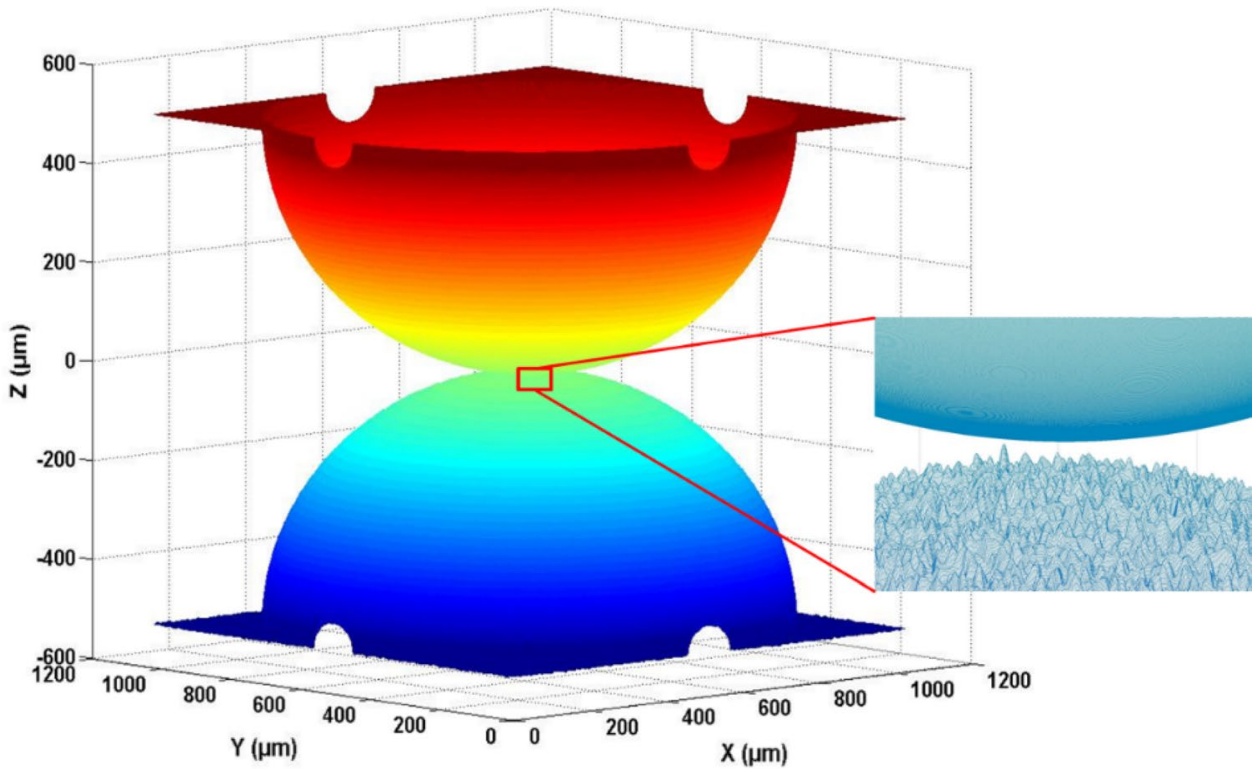


to test the accuracy of the numerical model prior to any simulations of rough surfaces and the results are plotted in Fig. 3.

Simulations are carried out for RMS roughness $\left(\mathrm{S}_{\mathrm{q}}\right)$ values of $0.1,0.2,0.5$ and $1 \mu \mathrm{m}$. Firstly, the simulations for pure elastic contacts are conducted. The results are plotted in Fig. 4, and suggest that an increase in the $\mathrm{S}_{\mathrm{q}}$ of the roughness of the surface shifts the normal force-displacement curve to the right as expected. For rougher surfaces, the highest asperity peaks carry the load and more compression is needed for achieving higher normal loads.

In contact of rough compressive surfaces, plastic deformation of the highest asperities readily occurs, if contact pressure exceeds the yield value, considering that the contact area is small. Thus pure elastic contact deformation of rough surfaces may not represent the correct stress fields. For this reason, the elastic-perfectly plastic deformation is used in this work and the results for different $S_{q}$ values are reported in Fig. 5. A small difference prevails as compared to the pure elastic simulations of Fig. 4. The difference increases as the surfaces become rougher due to plastic deformation.

\section{Modelling of bulk compression}

In a number of research and industrial application fields such as chemical and process engineering [13], civil engineering [22], and agricultural engineering [21], the behaviour of bulk particulate materials subjected to compression is of great interest.

In geotechnical engineering, soil-footing interactions or shallow footing is one of longest-dated topics with

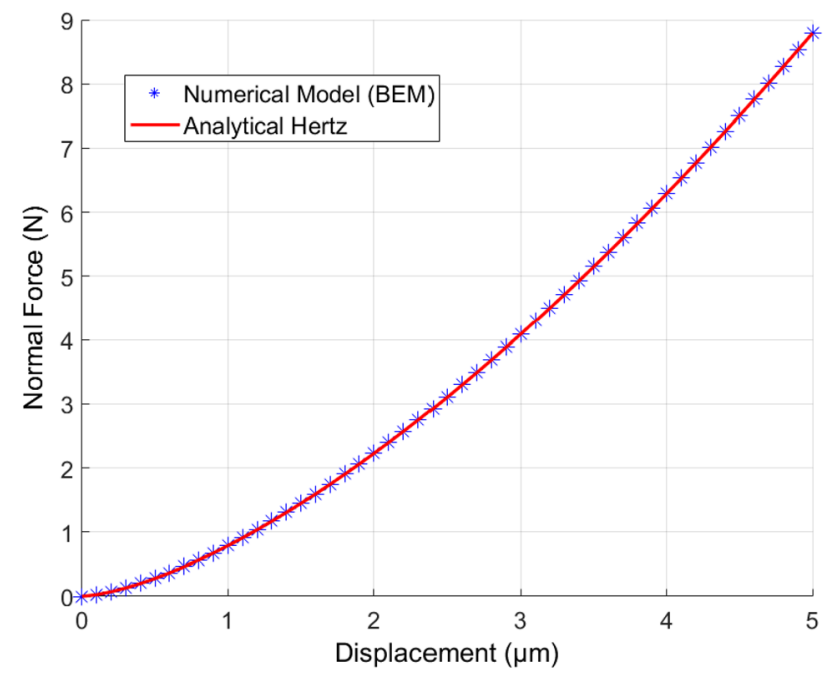

Fig. 3 Comparison between purely elastic BEM numerical model and the analytical Hertzian solution for two smooth particles in contact $\left(\mathrm{E}_{1}=\mathrm{E}_{2}=70 \mathrm{GPa}\right)$

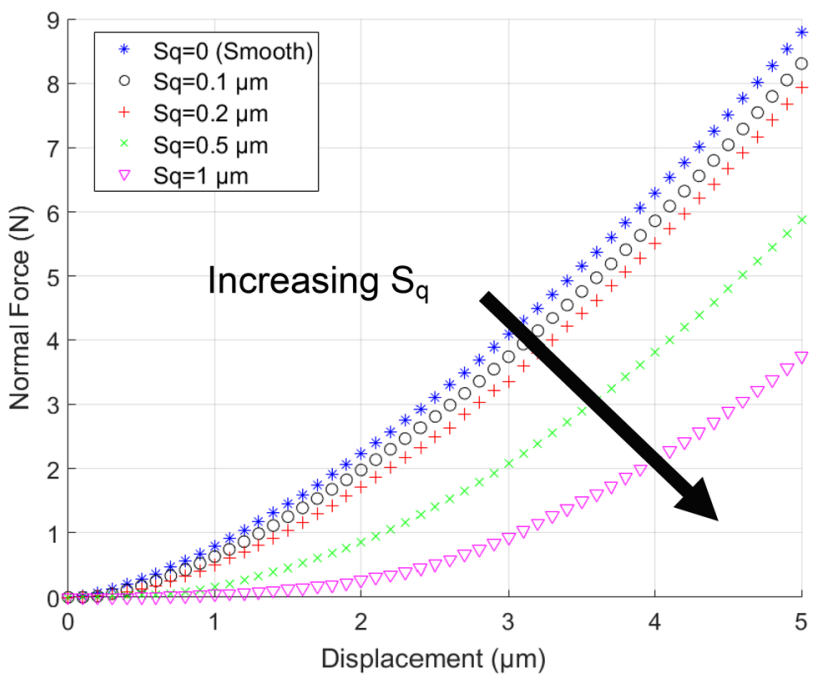

Fig. 4 Normal force-displacement for pure elastic contact of rough surfaces (arrow shows the increase in the $\mathrm{S}_{\mathrm{q}}$ value, $\mathrm{E}_{1}=\mathrm{E}_{2}=70 \mathrm{GPa}$ )

permanent relevance to practice [29]. This topic has received increasing attention in recent years, in particular for the design of offshore structures (e.g. [9, 17]). Previous DEM modelling reported by Gabrieli et al. [6] and Fu et al. [5] highlights the importance of the coefficient of friction on structural stiffness. However, there is no report on the role of surface roughness. Here, the effect of particle surface roughness on the bulk deformation mechanism of shallow footing is investigated using a BEM-DEM approach.

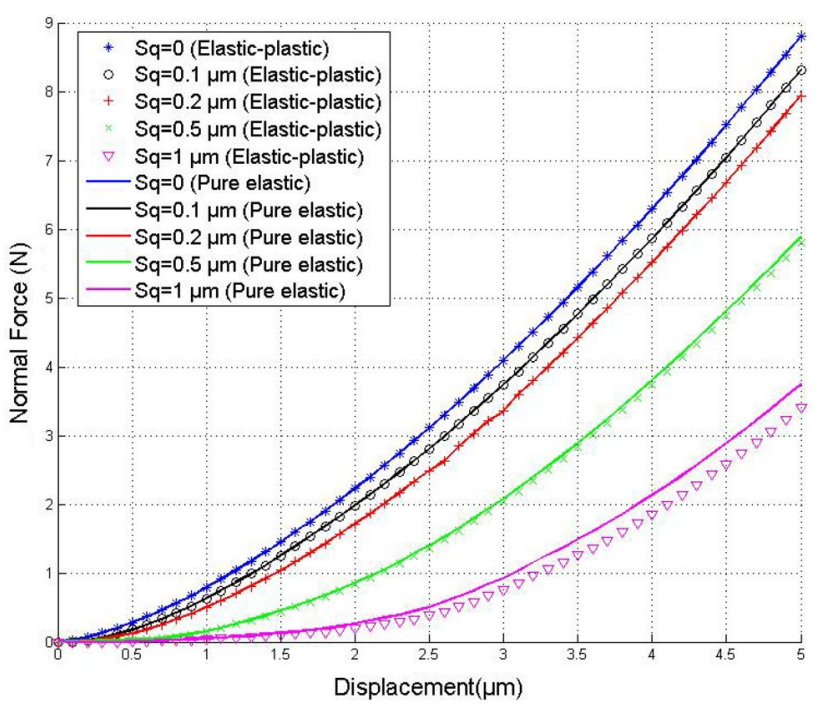

Fig. 5 Normal force-displacement for elastic-perfectly plastic contact of rough surfaces (scattered points are elastic-plastic simulations and lines are pure elastic, $\mathrm{E}_{1}=\mathrm{E}_{2}=70 \mathrm{GPa}$ and $\mathrm{H}=6 \mathrm{GPa}$ ) 


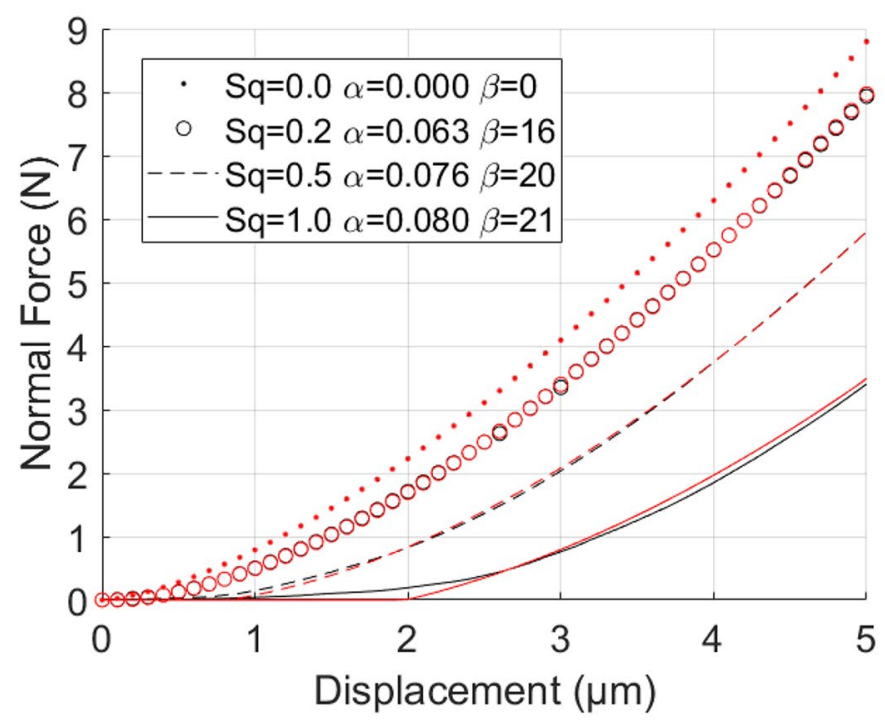

(a)

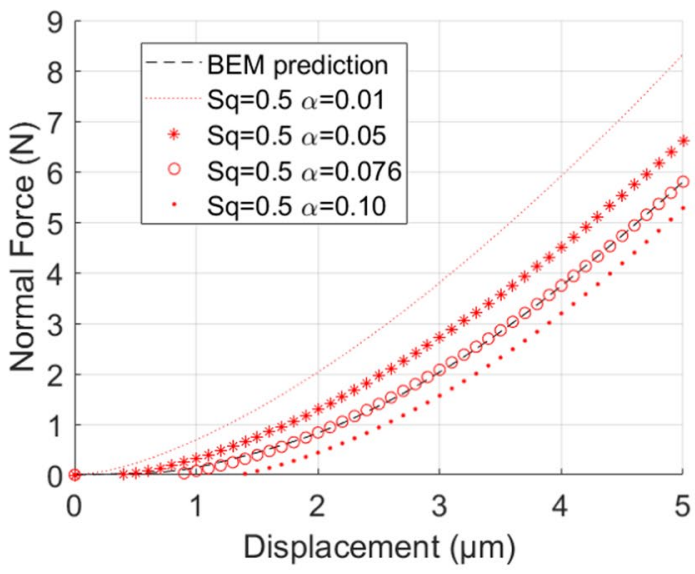

(b)

Fig. 6 a Comparison between the prediction of BEM (black lines and markers) and Eq. 6 (red line and markers); b sensitivity of the prediction to $\alpha$ value when $\beta$ is constant and equal to 20 for the case of

EDEM software package (DEM-Solutions, Edinburgh, UK) is used for DEM simulation. For contact deformation, an Application Programme Interface (API) is used employing standard $\mathrm{C}++$ scripting. The direct implementation of BEM algorithm in DEM requires calculation of contact forces at each time step, so it is computationally very expensive. Therefore, an alternative approach is adopted in which a relationship between force and displacement is first developed and implemented taking account of the effect of roughness on particle dynamics. In this study, the Hertz model has been modified to account for elastic-plastic deformation of roughness.

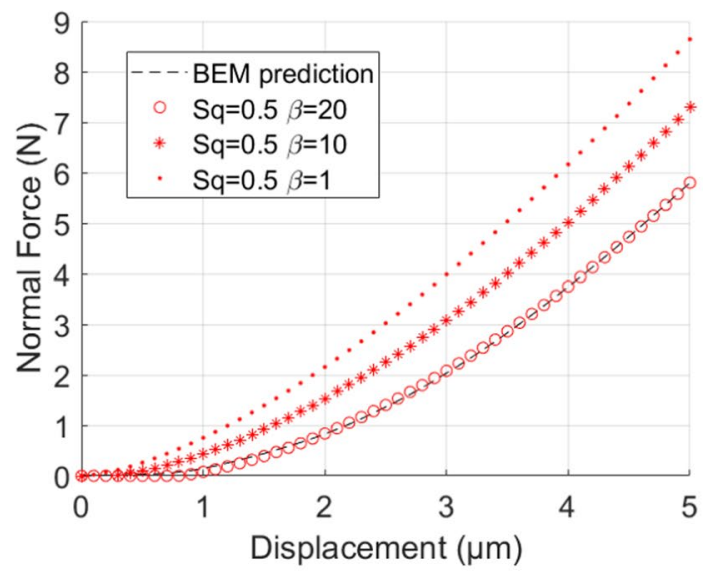

(c)

$\mathrm{S}_{\mathrm{q}}=0.5$; c sensitivity of the prediction to $\beta$ value when $\alpha$ is constant and equal to 0.076 for the case of $\mathrm{S}_{\mathrm{q}}=0.5$. (Color figure online)

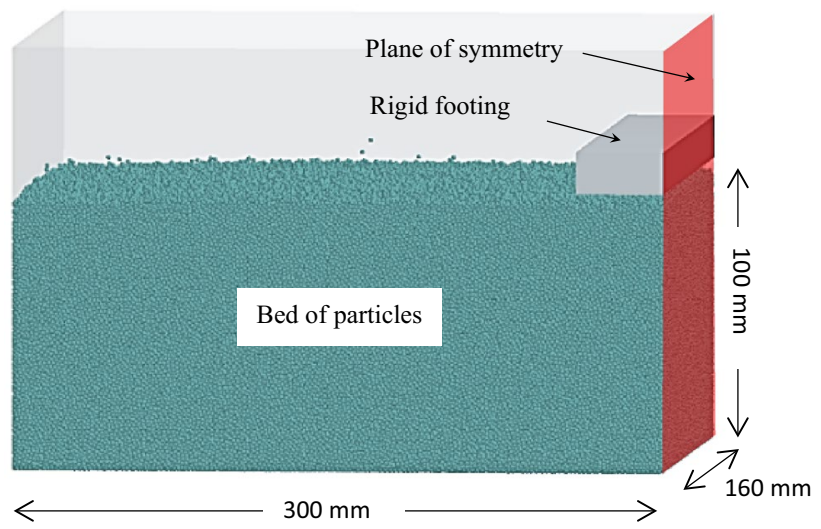

Fig. 7 The layout of the model showing the plane of symmetry 
Table 1 Particle properties in the simulation

\begin{tabular}{ll}
\hline Parameters & Value \\
\hline Particle density $\left[\rho\left(\mathrm{kg} / \mathrm{m}^{3}\right)\right]$ & 2500 \\
Young's modulus $[E(\mathrm{GPa})]$ & 70 \\
Poisson's ratio $(\nu)$ & 0.25 \\
Restitution coefficient $(e)$ & 0.5 \\
Coefficient of friction $(\varphi)$ & $0.3,0.5,0.9$ \\
\hline
\end{tabular}

\subsection{Customised contact model}

Based on Hertz anslysis [16], Eq. 5 provides a relationship between normal force $\left(F_{N}\right)$ and displacement $(\delta)$ for two smooth identical spheres in contact.

$$
F_{N}=\frac{4}{3} E^{*} \cdot R^{\left(\frac{1}{2}\right)} \cdot \delta^{\left(\frac{3}{2}\right)}
$$

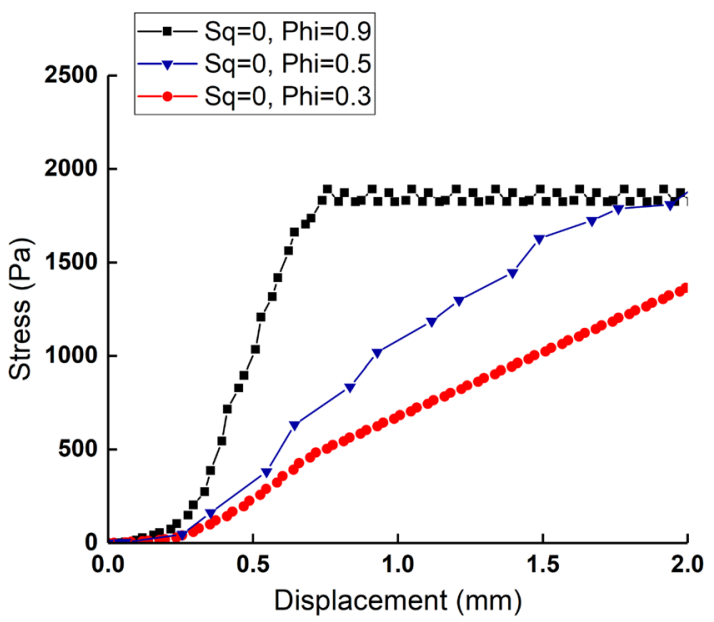

(a)

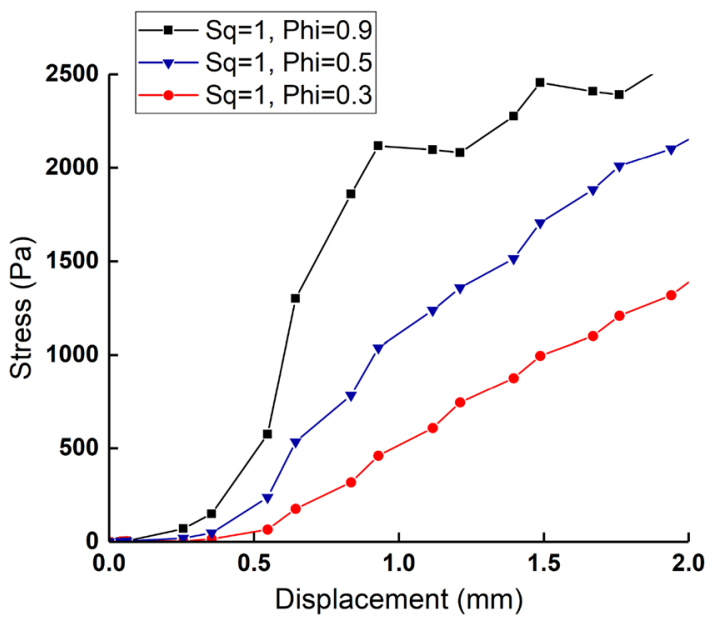

(c) where $E^{*}$ is the effective contact Young's modulus given by $E^{*}=E /\left(1-v^{2}\right)$ and $R$ is the reduced radius $\left(\frac{1}{R}=\frac{1}{R_{1}}+\frac{1}{R_{2}}\right)$, $E$ is the elastic modulus and $\nu$ is Poisson's ratio of the rough surface.

As reported in the literature and seen in the previous section, the rough surfaces show Hertzian response after a threshold normal force value [10]. For a given normal displacement, the normal load is lower for a rough particle than a smooth one. This difference in load (between smooth and rough cases) increases by increasing the normal displacement and becomes constant after the threshold force value. This trend can be represented by adding an error function to the normal force-displacement relationship (Eq. 6). As the value of surface roughness changes due to compression, a non-dimensional parameter, $\frac{\delta}{S_{q}^{*}}$, is used in the error function. Greenwood and Tripp [10] proposed the following

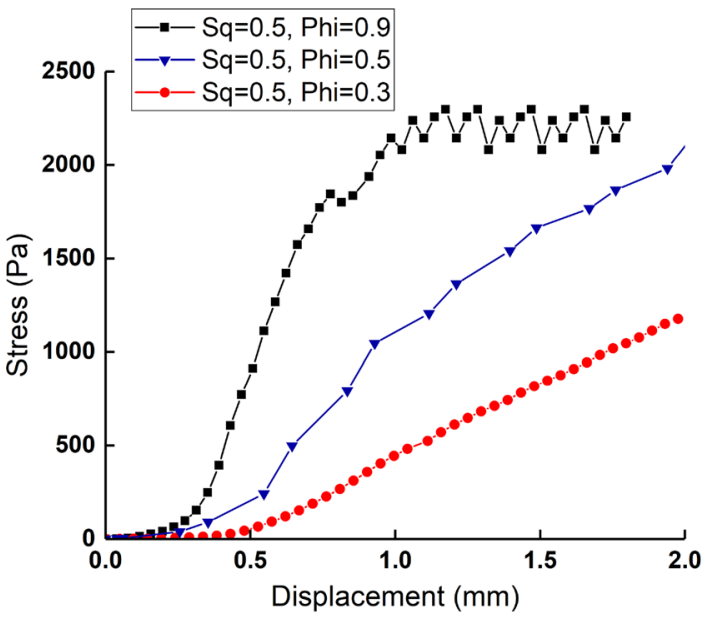

(b)

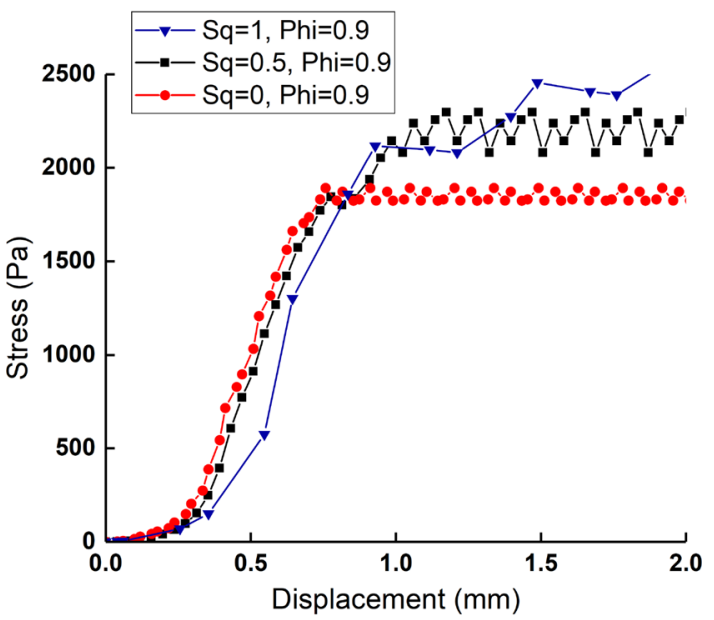

(d)

Fig. 8 Stress-displacement curve of footing on bed of particles with constant $S_{q}$ and varying coefficient of friction (a-c), and varying $S_{q}$ and constant coefficient of friction $(\mathbf{d})$ 
relationship to approximate the threshold normal contact force, $F_{N}=S_{q}^{*} E^{*} \sqrt{2 R^{*} S_{q}^{*}}$. In Eq. 6, two constants $\alpha$ and $\beta$ are introduced into the model, where their values are obtained by comparing with BEM calculation. In this approach, the effect of roughness on normal load can be implemented with high efficiency. The modified normal force-displacement relationship is as follows:

$F_{N}=\frac{4}{3} E^{*} \cdot \sqrt{R^{*}} \cdot \delta^{\left(\frac{3}{2}\right)}-\beta S_{q}^{*} E^{*} \sqrt{R^{*} S_{q}^{*}} \operatorname{erf}\left(\alpha \frac{\delta}{S_{q}^{*}}\right) \quad F N \geq 0$
The prediction of Eq. 6 for different values of $\mathrm{S}_{\mathrm{q}}$ is compared with the BEM results in Fig. 6a. The values of $\alpha$ and $\beta$ are listed in legend of Fig. 6 a for four roughness values with the coefficient of determination $R^{2}=99 \%$. Figure $6 \mathrm{~b}$ shows the effect of $\alpha$ when all other variables are constant. It can be seen, $\alpha$ controls the so called 'initial plasticity'. Figure $6 \mathrm{c}$ presents the effect of $\beta$ on force-displacement relationship. $\beta$ can change the slope of the force-displacement curve. This model is implemented in EDEM and is verified in the next section.

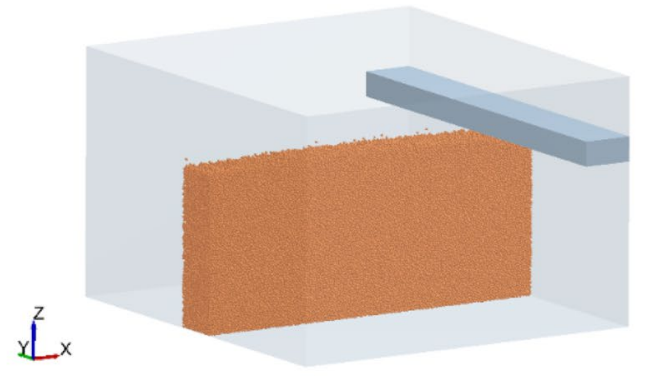

(a)

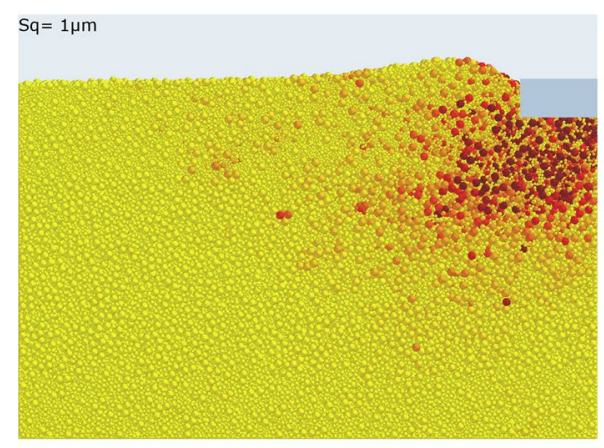

(b)

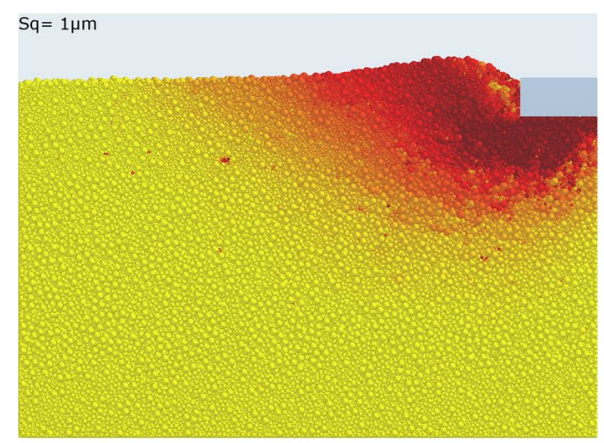

(d)

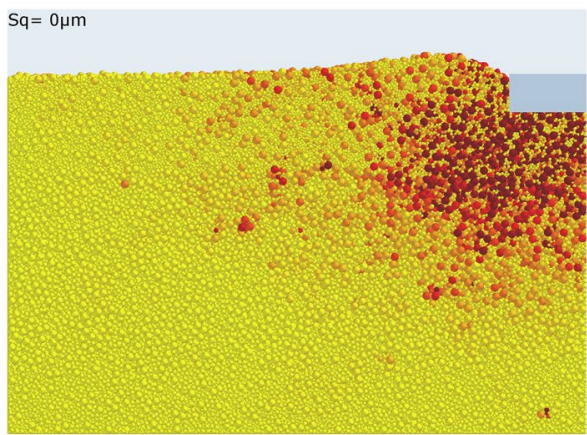

(c)

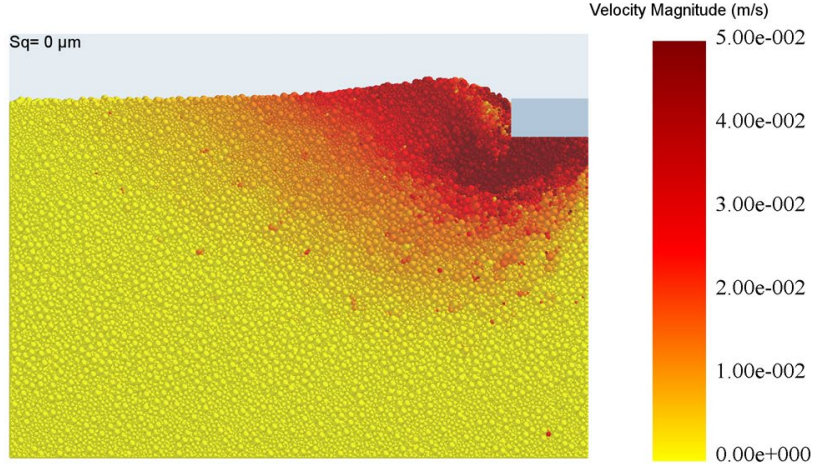

(e)
Total Force Magnitude (N)

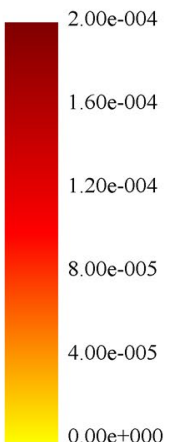

$0.00 \mathrm{e}+000$ 

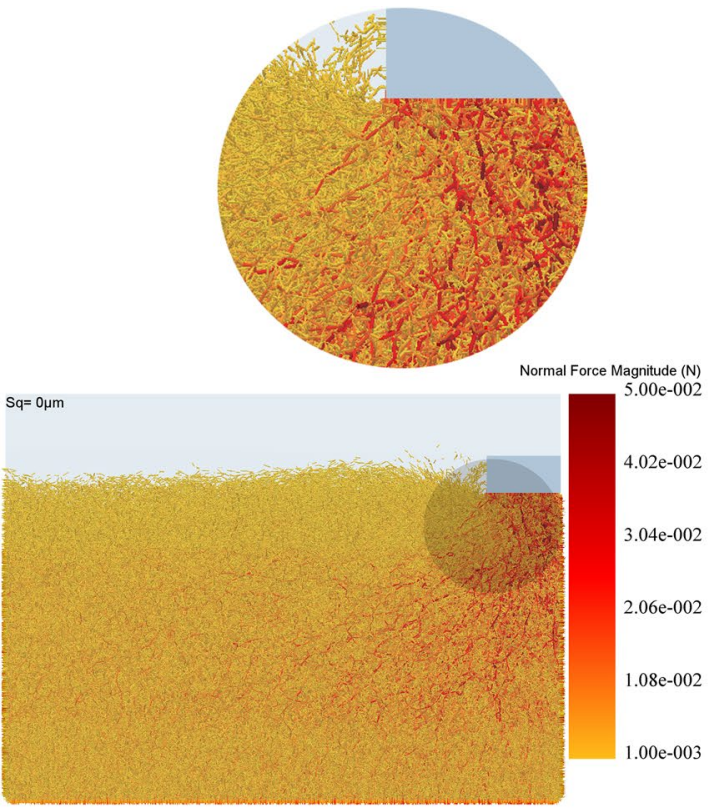

(a)

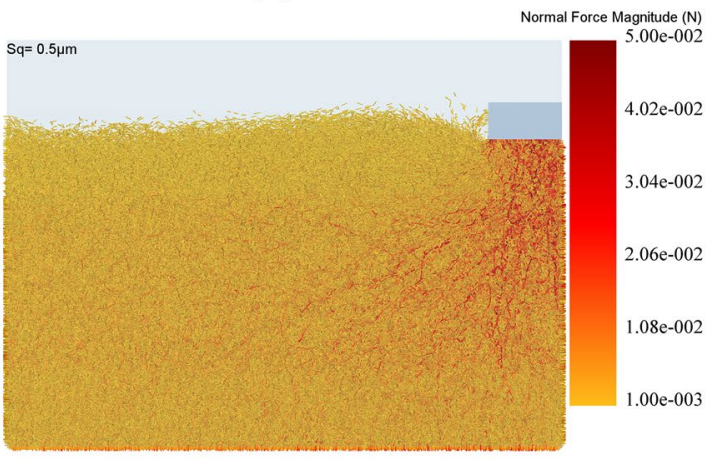

(c)

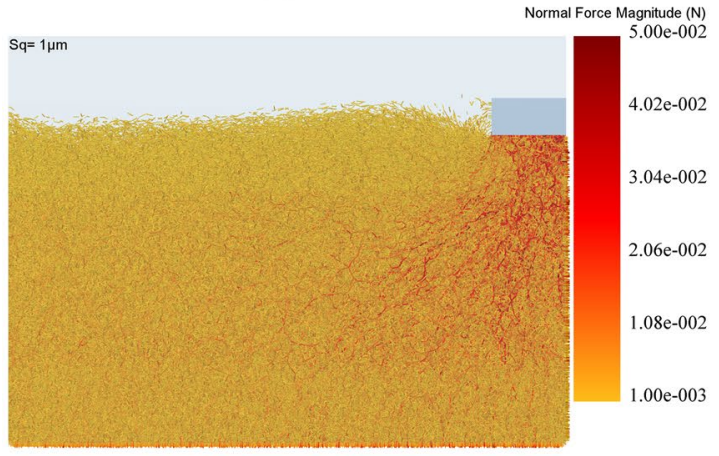

(e)

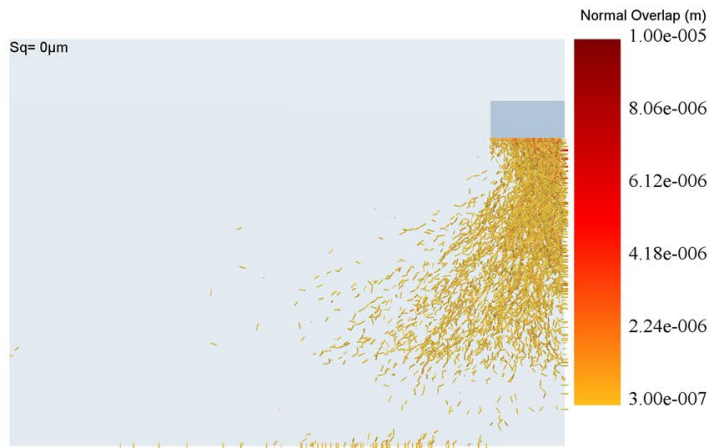

(b)

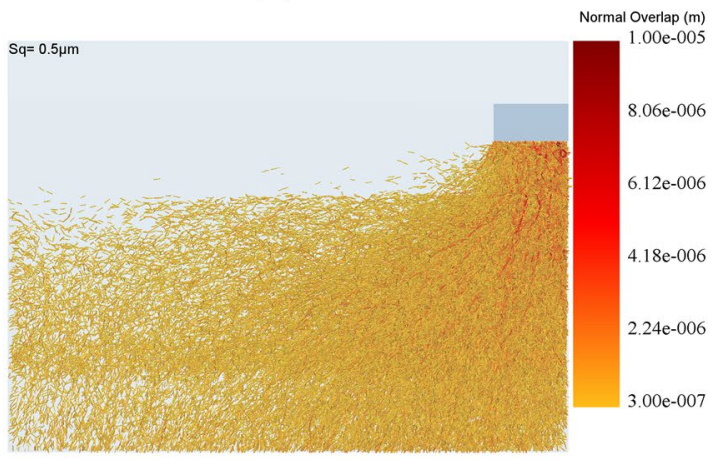

(d)

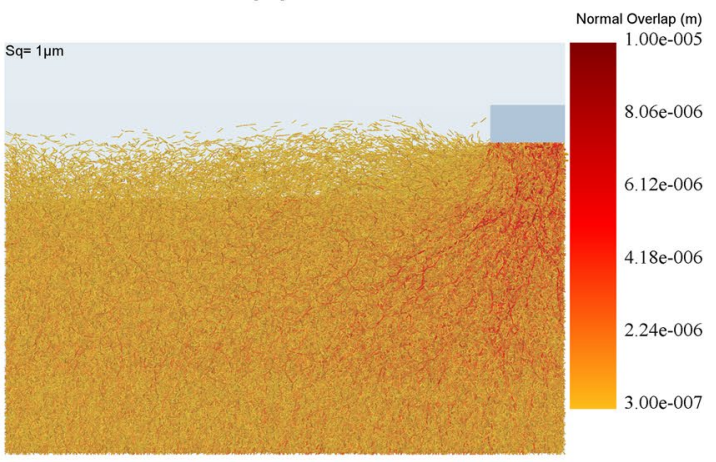

(f)

Fig. 10 Normal contact force network and contact deformation for particles with surface roughness of a, b $S_{q}=0 ; \mathbf{c}, \mathbf{d ~} S_{q}=0.5 ; \mathbf{e}, \mathbf{f} S_{q}=1 \mu m$ $(\varphi=0.5)$

\subsection{Numerical simulation of penetration of a rigid footing into a granular bed}

Following Nadimi et al. [19], a box with internal dimension of $300 \mathrm{~mm} \times 160 \mathrm{~mm} \times 100 \mathrm{~mm}$ is used as shown in Fig. 7. 1.7 million particles are pluviated under gravity into half of the box and allowed to settle. Using symmetry reduces the computational expense. The particle size distribution consists of $20 \%$ of $0.78 \mathrm{~mm}$ diameter, $50 \%$ of $1.3 \mathrm{~mm}$, and $30 \%$ of 


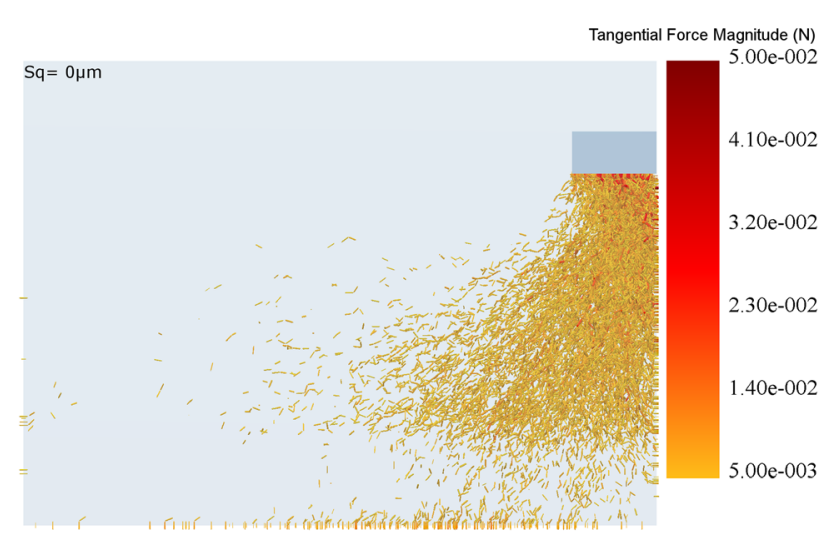

(a)

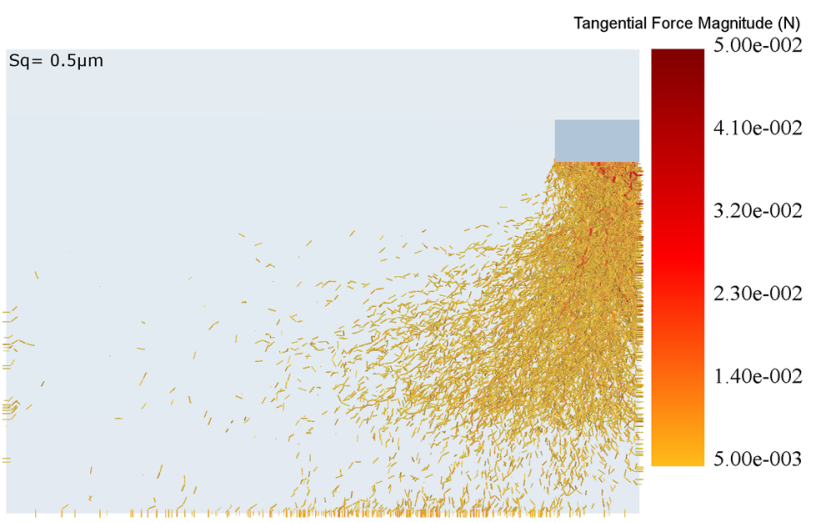

(c)

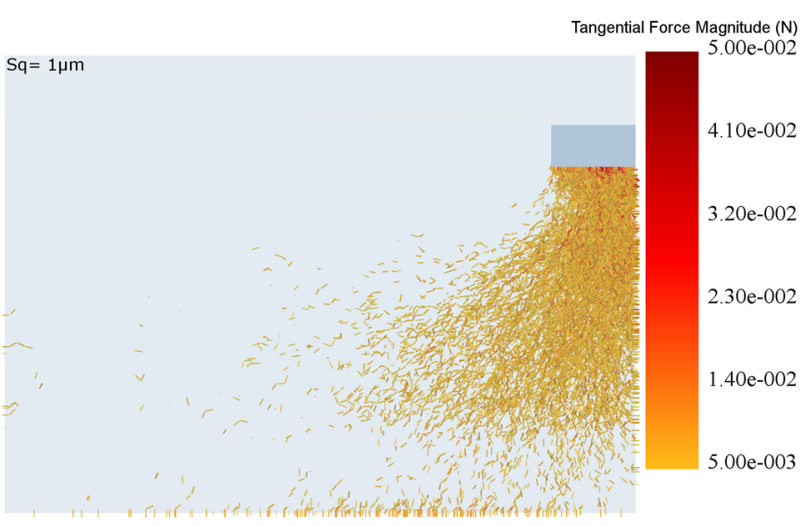

(e)

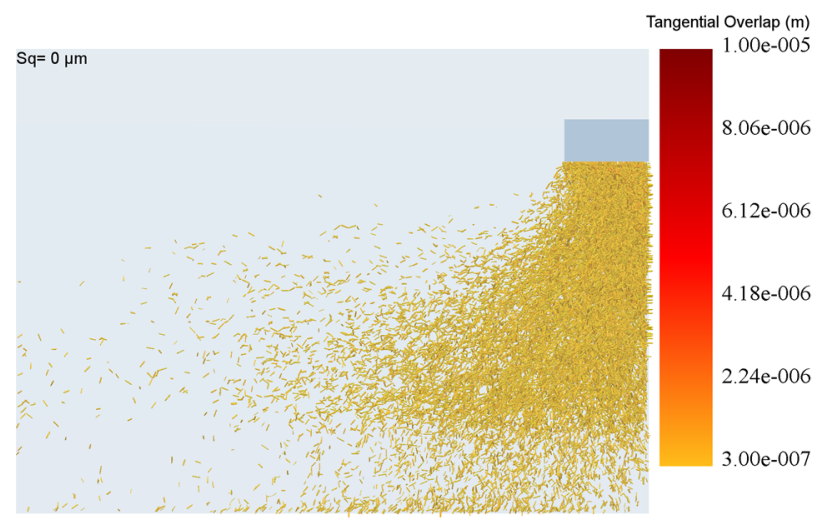

(b)

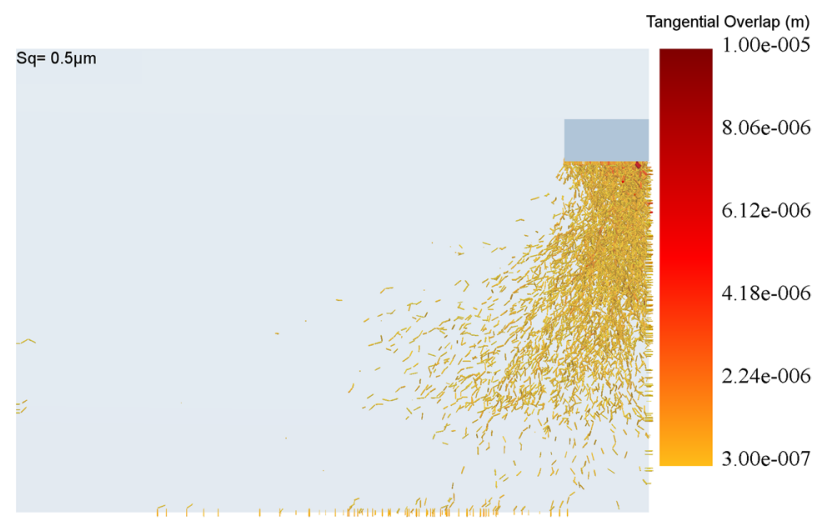

(d)

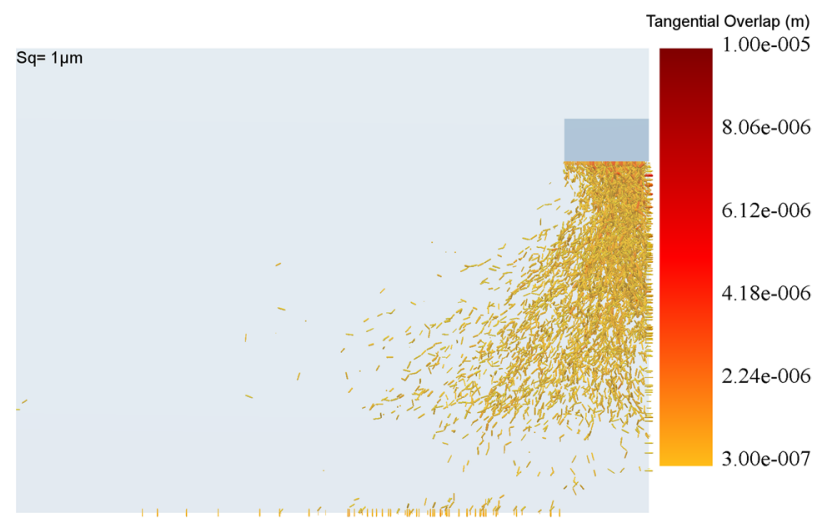

(f)

Fig. 11 Tangential force network and contact slip for particles with surface roughness of $\mathbf{a}, \mathbf{b} \mathrm{S}_{\mathrm{q}}=0 ; \mathbf{c}, \mathbf{d} ; \mathrm{S}_{\mathrm{q}}=0.5 ; \mathbf{e}, \mathbf{f} \mathrm{S}_{\mathrm{q}}=1 \mu \mathrm{m}(\varphi=0.5)$

$2.1 \mathrm{~mm}$ particles following the experimental data of Nadimi et al. [19]. A rigid footing with the thickness of $10 \mathrm{~mm}$, the width of $40 \mathrm{~mm}$ and the length of $160 \mathrm{~mm}$ is placed on the particles bed to represent plane strain condition. Regarding boundary effects, experimental and finite element simulations reported by Nadimi et al. [19] show that zone of maximum deformation is within the boundary of the model. The footing is pressed into the bed for a total displacement of $4 \mathrm{~mm}$ at quasi-static condition $(0.001 \mathrm{~mm} / \mathrm{s})$. The physical and mechanical properties of particles are reported in the Table 1. In total, nine simulations are run with particle roughness values, i.e. $\mathrm{S}_{\mathrm{q}}=0,0.5$ and $1 \mu \mathrm{m}$ and coefficient of friction values of $\varphi=0.3,0.5$ and 0.9 . 


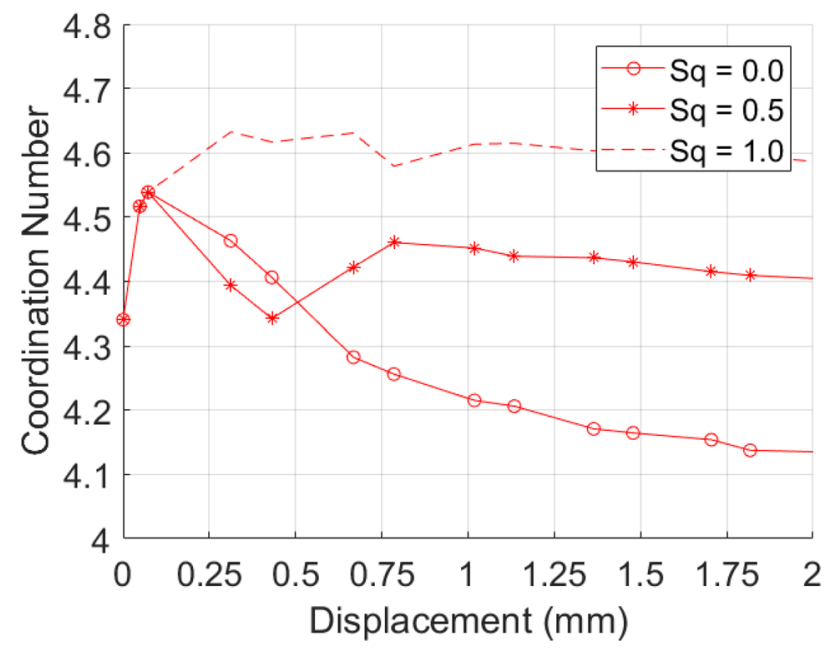

Fig. 12 Evolution of coordination number for three different surface roughness $(\varphi=0.5)$

\subsection{Results}

In this study, the normal stiffness has only been modified for rough particles. The effect of roughness on tangential stiffness is more complicated and yet to be developed. Therefore, to investigate the effect of inter-particle friction on bulk behaviour, Fig. $8 \mathrm{a}-\mathrm{c}$ show the stress-displacement curves when the coefficient of friction is varied and particle roughness is kept constant. There is a general agreement between all the three Figures that the increase in coefficient of friction significantly increases the stiffness of the bed.

Figure 8d presents the macroscopic stress-displacement curves when the coefficient of friction is constant $(\varphi=0.9)$ and particle roughness is varied. The capacity of the bed increases with increase in particle surface roughness. For a given set of condition, there exists a threshold, in most cases, above which the stress no longer increases further. This is due to particle rearrangement, i.e. sliding and rolling, in the shear band formed. This threshold is controlled by particle surface roughness and inter-particle friction (Fig. 8).

In order to compare the deformation mechanism of the particulate bed for different roughness values, a $20 \mathrm{~mm}$ thick slice in the middle of the bed is selected to avoid the boundary effects as shown in Fig. 9a. The magnitudes of the total force experienced by particles are compared in Fig. 9b, c for the cases of $S_{q}=0$ and $S_{q}=1 \mu \mathrm{m}$, respectively. It can be seen that for rough particles the load is more localised/less distributed than smooth particles over the sheared volume. The velocity magnitudes of the particles at a given time, loading stage, are shown in Fig. 9d, e. The active zone of deformation can be clearly seen, where differences prevail between smooth and rough particles.
The normal contact force and associated magnitude together with contact deformation are illustrated in Fig. 10. This is obtained by drawing the contact vectors between particles and colouring them according to their magnitude of force or overlap. Normal force legend is limited to the range from 1 to $50 \mathrm{mN}$ and normal overlap legend is limited to 2 to $10 \mu \mathrm{m}$ for the sake of comparison. The effect of particle roughness on contact deformation can be clearly seen in these figures. Figure 10b shows contact deformation of smooth particles. By increasing the particle roughness, more particles experience large contact deformation and more wide spread (Fig. 10d, f). In contrast to the trend observed in normal contact deformation, the tangential slip or overlap is reduced by increasing the particle roughness (Fig. 11b, $\mathrm{d}, \mathrm{f})$. This shows that the smoother particles slide easier on each other. The tangential force network does not show any notable differences for the three surface roughness values (Fig. 11a, c, e). This is considered to result from not taking into account the influence of interlocking on tangential traction, a case to be considered in the next step.

Figure 12 shows the effect of particle roughness on the evolution of the coordination number. Rough particles have a larger average coordination number than smooth particles during the compression. Furthermore, the coordination number decreases on penetration for the smooth case due to dilation around the footing zone. It can be concluded that an increase in the bearing capacity of the footing for the rough particles is related to the larger number of inter-particle contacts around the footing.

\section{Conclusions}

A novel approach for accounting elasto-plastic deformation of rough surface is presented by coupling BEM derived contact behaviour and DEM modelling for predicting bulk compression behaviour of a penetrating footing. In this framework, the effect of surface roughness of a particle on its contact interaction is quantified. A general contact model is proposed by considering the surface roughness in normal contact deformation behaviour. The model is implemented in EDEM commercial software to simulate the bulk behaviour. The developed model is employed to investigate the effect of particle roughness on bulk compression mechanism. In micromechanical investigations, it is shown that the spatial distribution of contact deformation increases in the normal direction, whilst in contrast the tangential slip reduces when increasing the particle surface roughness. The bed capacity increases for rough particles. This is attributed to a larger number of contacts present around the footing as it penetrates into the bed of rough particles. The proposed BEM-DEM framework can be employed for any material of 
interest with different surface roughness undergoing elastoplastic deformation.

Acknowledgements This work is supported by the Engineering and Physical Sciences Research Council (Grant Number EP/001766/1) as a part of 'Friction: The Tribology Enigma' Programme Grant (www. friction.org.uk), a collaboration between the Universities of Leeds and Sheffield.

\section{Compliance with ethical standards}

Conflict of interest The authors declare that they have no conflict of interest.

Open Access This article is distributed under the terms of the Creative Commons Attribution 4.0 International License (http://creativeco mmons.org/licenses/by/4.0/), which permits unrestricted use, distribution, and reproduction in any medium, provided you give appropriate credit to the original author(s) and the source, provide a link to the Creative Commons license, and indicate if changes were made.

\section{References}

1. Cavarretta I, Coop M, O'Sullivan C (2010) The influence of particle characteristics on the behaviour of coarse grained soils. Géotechnique 60(6):413-423

2. Chilamakuri SK, Bhushan B (1998) Contact analysis of nonGaussian random surfaces. Proc the Inst Mech Eng Part J J Eng Tribol 212(1):19-32

3. Cundall PA, Strack OD (1979) A discrete numerical model for granular assemblies. Géotechnique 29(1):47-65

4. Ferellec JF, McDowell GR (2010) A method to model realistic particle shape and inertia in DEM. Granular Matter 12(5):459-467

5. Fu Z, Chen S, Liu S (2016) Discrete element simulations of shallow plate-load tests. Int J Geomech 16(3):04015077

6. Gabrieli F, Cola S, Calvetti F (2009) Use of an up-scaled DEM model for analysing the behaviour of a shallow foundation on a model slope. Geomech Geoeng Int J 4(2): 109-122

7. Ghanbarzadeh A, Wilson M, Morina A, Neville A (2016) A new strategy for the relative movement of rough surfaces in contact using a boundary element method. Eur J Comput Mech 25(4):309-328

8. Ghanbarzadeh A, Wilson M, Morina A, Dowson D, Neville A (2016) Development of a new mechano-chemical model in boundary lubrication. Tribol Int 93:573-582

9. Govoni L, Gourvenec S, Gottardi G (2011) A centrifuge study on the effect of embedment on the drained response of shallow foundations under combined loading. Géotechnique 61(12):1055-1068

10. Greenwood JA, Tripp JH (1967) The elastic contact of rough spheres. J Appl Mech 34(1):153-159

11. Greenwood JA, Johnson KL, Matsubara E (1984) A surface roughness parameter in Hertz contact. Wear 100(1-3):47-57

12. Hare C, Ghadiri M (2013) The influence of aspect ratio and roughness on flowability. AIP Conf Proc 1542(1):887-890

13. Hassanpour A, Ghadiri M (2004) Distinct element analysis and experimental evaluation of the Heckel analysis of bulk powder compression. Powder Technol 141(3):251-261
14. Houlsby GT (2009) Potential particles: a method for modelling non-circular particles in DEM. Comput Geotech 36(6):953-959

15. Hu YZ, Tonder K (1992) Simulation of 3-D random rough surface by 2-D digital filter and Fourier analysis. Int J Mach Tools Manuf 32(1-2):83-90

16. Johnson KL (1987) Contact mechanics. Cambridge University Press, Cambridge

17. Lau CK, Bolton MD (2011) The bearing capacity of footings on granular soils. II: Experimental evidence. Géotechnique 61(8):639-650

18. Majmudar TS, Behringer RP (2005) Contact force measurements and stress-induced anisotropy in granular materials. Nature 435(7045):1079-1082

19. Nadimi S, Fonseca J, Barreto D, Taylor RN (2016) A new approach to investigate the particle size effects in centrifuge modelling. Paper presented at the The 3rd European conference on physical modelling in geotechnics, 1-3 Jun 2016, Nantes, France

20. Nadimi S, Fonseca J (2018) A micro finite-element model for soil behaviour. Géotechnique 68(4):290-302

21. Nona KD, Lenaerts B, Kayacan E, Saeys W (2014) Bulk compression characteristics of straw and hay. Biosyst Eng 118:194-202

22. Oda M, Koishikawa I (1979) Effect of strength anisotropy on bearing capacity of shallow footing in a dense sand. Soils Found 19(3): 15-28

23. Otsubo M, O'Sullivan C, Hanley KJ, Sim WW (2017) The influence of particle surface roughness on elastic stiffness and dynamic response. Géotechnique 67(5):452-459

24. Pohlman NA, Severson BL, Ottino JM, Lueptow RM (2006) Surface roughness effects in granular matter: influence on angle of repose and the absence of segregation. Phys Rev E 73(3):031304

25. Powrie W, Ni Q, Harkness RM, Zhang X (2005) Numerical modelling of plane strain tests on sands using a particulate approach. Géotechnique 55(4):297-306

26. Sahlin F, Larsson R, Almqvist A, Lugt PM, Marklund P (2010) A mixed lubrication model incorporating measured surface topography Part 1: theory of flow factors. Proc Inst Mech Eng Part J J Eng Tribol 224(4):335-351

27. Santamarina C, Cascante G (1998) Effect of surface roughness on wave propagation parameters. Géotechnique 48(1):129-136

28. Sharifipour M, Dano C (2006) Effect of grains roughness on waves velocities in granular packings. In: Proceedings of the 1st Euro Mediterranean conference on advances on geomaterials and structure. Hammamet, pp. 123-128

29. Terzaghi K, Peck RB (1948) Soil mechanics in engineering practice, 1st edn. Wiley, New York

30. Vakis AI, Yastrebov VA, Scheibert J, Minfray C, Nicola L, Dini D, Almqvist A, Paggi M, Lee S, Limbert G, Molinari JF (2018) Modeling and simulation in tribology across scales: an overview. Tribol Int 125:169-199

31. Wilson R, Dini D, Van Wachem B (2017) The influence of surface roughness and adhesion on particle rolling. Powder Technol 312:321-333

32. Zhao T, Feng YT (2018) Extended Greenwood-Williamson Models for Rough Spheres. J Appl Mech 85(10):101007

Publisher's Note Springer Nature remains neutral with regard to jurisdictional claims in published maps and institutional affiliations. 